\title{
Qualification of Cluster Header and Cluster Member in Wireless Sensor Networks
}

\author{
EunHwa Kim
}

\begin{abstract}
Many researches have been proposed for efficiency of data transmission from sensor nodes to sink node for energy efficiency in wireless sensor networks. Among them, cluster-based methods have been preferred In this study, we used the angle formed with the sink node and the distance of the cluster members to calculate the probability of cluster head. Each sensor node sends measurement values to header candidates, and the header candidate node measures the probability value of the header with the value received from its candidate member nodes. To construct the cluster members, the data transfer direction is considered. We consider angle, distance, and direction as cluster header possibility value. Experimental results show that data transmission is proceeding in the direction of going to the sink node. We calculated and displayed the header possibility value of the neighbor nodes of the sensor node and confirmed the candidates of the cluster header for data transfer as the value. In this study, residual energy amount of each sensor node is not considered. In the next study, we calculate the value considering the residual energy amount of the node when measuring the header possibility value of the cluster.
\end{abstract}

Keywords : Wireless Sensor Networks, Clustering, Cluster Head, Cluster Member, Energy Efficiency

\section{INTRODUCTION}

$\mathrm{A}_{\mathrm{s} \text { wireless sensor networks have been utilized in various }}$ fields, people's lives have become more convenient and beneficial. Especially, in a region where people cannot reach easily, the wireless sensor network can be used to instantly process various data, and response in a dangerous situation can be accelerated.

With the advancement of hardware technology, the size of wireless sensors has become smaller and more sophisticated. Despite these advances, there are still battery limitations in wireless sensor networks. The use of efficient energy in an environment where batteries cannot be recharged is one of the most important elements of wireless sensor networks. Various methods for efficient energy have been proposed [1-3]. Among them, the clustering technique is to group the individual sensor nodes locally to transmit data more efficiently. A number of clustering techniques have been proposed since $\mathrm{LEACH}$, and this paper also proposes a more efficient method for cluster configuration.

In this paper, we consider the residual energy amount and member node elements in the data direction for the qualification factor of the cluster headers. For each sensor node, the value is calculated so that the probability of being

Revised Manuscript Received on July 22, 2019.

EunHwa Kim, Department of Computer Science, YongIn University, Republic of Korea, Email: ehkimanna@yongin.ac.kr selected as the header becomes higher as the closer to the optimal header value. Assuming that each sensor node transmits data on a hop-based basis, the sensor node closest to the sink node in the communication radius becomes the optimal relaying node. The cluster member also considers only the sensor nodes farther from the sink in terms of data transmission direction within the communication radius of the cluster header. Clustering proposed in this paper is analyzed to minimize the transmission path to the sink node. In order to prevent the direction of data transfer in the cluster from being in the reverse direction to the sink, the angle constraint was given in the cluster header when selecting members. The probabilistic method was used for the selection of the headers, and the probability values were determined considering the distance to the headers and the angle of the sink node and the header, without regard to all neighbor nodes within the communication radius. Simulation results show that it is possible to reduce the propagation path length through the angle of the cluster header in the energy efficient clustering method, which is longer than the optimum path length directly transmitted.

The composition of this paper is as follows. In Section 2, we discuss related works. Section 3 describes the proposed method for the selection of elements and members of the headers in relation to the cluster configuration. We simulate the proposed method, show various data in Section 4, and finally conclude in Section 5

\section{RELATED WORKS}

Cluster configuration has been approached in a variety of ways. The cluster-based proposed routing schemes can be classified as shown in Figure 1 [7].

The cluster header selection method has been proposed variously [4-6, 8-11]. They may be pre-selected in the center or may be elected in a decentralized manner. When considering a very large area, an autonomous selection method is recommended rather than a centralized method. The autonomous selection scheme may be a position-based scheme or a random scheme.

The probability of header increases as the amount of residual energy increases and the number of neighboring nodes increases. Neighbor node is all nodes within the communication radius of the header. In some cases, the size of the cluster can be expanded. When the data transferred directly in the cluster, the energy consumption increases exponentially. When the multi-hop is used intra-cluster, the routing method in the cluster again needs. 
LEACH is the first paper to introduce cluster-based routing concepts [8]. In LEACH, the header is selected by a random probability scheme based on the amount of energy remaining and the number of neighboring nodes, and the header directly communicates with the sink node. The following equation 1 and 2 are the amount of data transfer energy used in LEACH. These values used in our simulations. Equation 1 is an equation for the amount of energy required to send data, and Equation 2 is an equation for the amount of energy required to receive data $[8,10]$. In the equation, $E_{\text {ele }}$ is $50 \mathrm{~nJ} / \mathrm{bit}$ and $\varepsilon_{a m p}$ is $100 \mathrm{pJ} / \mathrm{bit} / \mathrm{m} 2$.

$E_{T X}(k, d)=E_{T X-E L E C}(k)+E_{T X-A M P}(k, d)=E_{\text {ele }} * k+\varepsilon_{\text {amp }} * k^{*} d^{2}$

$E_{R X}(k, d)=E_{R X-E L E C}(k)=E_{\text {ele }} * k$

In addition to LEACH, GAF to select the header at the lattice location and PEGASIS to construct the node in the chain form have been proposed [10-13].

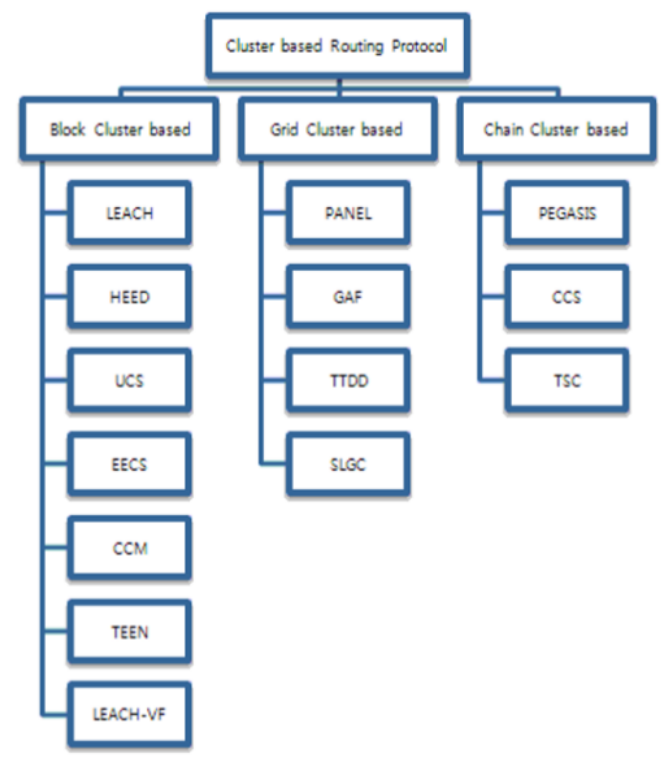

Fig 1. Cluster based routing methods

\section{CLUSTER HEADER AND MEMBER}

The most important factor in the cluster configuration is how to select the cluster header and how to configure cluster members [14-15]. In this paper, we select cluster headers randomly based on probabilities. The higher the amount of residual energy and the more cluster members, the higher the probability of headers. In general, the configuration of a cluster member extends to one hop or two hops centered on the communication radius. When considering cluster members, we do not consider all sensor nodes within the communication radius. Since the data is transmitted in the sink direction, the relationship between the cluster member and the header is selected in consideration of the data transmission direction.

In the case of connecting to the sink node by multi-hop, the forwarding node going to the optimum shortest path becomes the closest node to the straight line connecting the sensor node and the sink node. The amount of energy consumed increases as the distance the sensor node sends data increases. Optimal forwarding nodes of energy consumption within the transmission range are nodes at the boundary.

As shown in Figure 2, let's select a cluster header from an arbitrary sensor node. A sensor node that is most suitable for data transmission in the direction of the sink node within the communication radius can be selected as the cluster header candidate. In Figure 2, sensor node A is closer to the sink node in the direction of data transfer, and sensor node B is located closer to the sink node in the communication radius. The optimal solution to find in this paper is the sensor node $\mathrm{C}$. We try to find a header node candidate that is closest to the straight line with sink node and that is the farthest within the transmission radius.

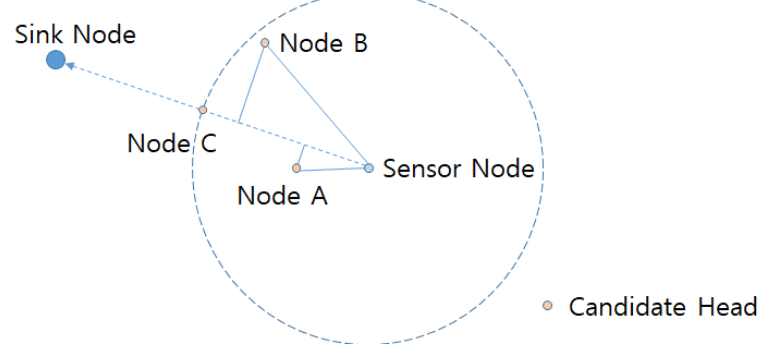

Fig 2. Factor of Cluster Header Selection

In this paper, we consider the positional factors as headers together with the residual energy amount as a requirement of header selection. Each sensor node computes the positional probability value of its own header as its header from the position information of surrounding nodes and the position of sink node.

A value that measures the distance from the line connecting the sensor node to the sink node can be calculated by the following $\cos \theta$ value. In the equation 3 , the coordinate value of the sink node is $\left(x_{S}, y_{S}\right)$, the coordinate value of the header candidate node is $\left(x_{H}, y_{H}\right)$, and the coordinate value of the sensor node is $\left(x_{N}, y_{N}\right)$.

$\cos \theta=\frac{\left(x_{S}-x_{H}\right)\left(x_{N}-x_{H}\right)+\left(y_{S}-y_{H}\right)\left(y_{N}-y_{H}\right)}{\sqrt{\left(x_{S}-x_{H}\right)^{2}+\left(y_{S}-y_{H}\right)^{2}} * \sqrt{\left(x_{N}-x_{H}\right)^{2}+\left(y_{N}-y_{H}\right)^{2}}}$

The closeness can be measured by the angle between the straight line connecting the sink node and the sensor node and the straight line connecting the header candidate node and the sensor node. The degree of distantness within the communication radius can be measured by the distance between the sensor node and the header candidate node.

The optimal header candidate node value is calculated by the following equation as the value of the position calculated by these two values. Of course, this value only calculates the pure position value excluding the residual energy amount.

Every sensor node is a candidate node that can be a header, and calculates the possibility value of the header based on the position value of its own neighbor node. Based on the calculated values, the header is selected in a random probability manner. In this paper, residual energy is excluded in order to consider only the position value purely. Equation (4) is a formula that each sensor node obtains a probability value that becomes a header by using the location information and the distance of neighboring nodes. The $P_{\text {Header }}$ value is a value between -1 and 0 , and the smaller the value, the higher the probability value as a header. 


$$
P_{\text {Header }}=\left\{\begin{array}{rr}
\sum \frac{\text { Distance (Node, Header) }}{\text { Transmission range }} * \cos \theta & \text { (if } \cos \theta<0) \\
0 & \text { (if } \cos \theta>0)
\end{array}\right.
$$

Each sensor node uses the neighbor node location information to collect the header probability value according to the distance and the direction to the sink node. At this time, the header possibility value of the neighboring nodes which are in the communication radius and to transmit data in the reverse direction is a positive value, and is calculated as a value of 0 in order not to reflect this. All the header possibility values from the neighbor nodes are accumulated so that the sensor node having the smallest value can be preferentially a header.

When the cluster header is determined, the sensor node broadcasts to the neighboring nodes, and the sensor nodes that have received the broadcast calculate the cluster configuration angle using the header position information, the sink node's position information, and the node's position information. Once determined, it becomes a cluster member and it no longer participates in a random way to become a cluster header. If a cluster member hears additional broadcasts from a cluster header that is closer to the sink during the cluster configuration period, it switches as a member of the cluster member to shorter cluster header.

As shown in Figure 3, a cluster member composed of cluster headers considers members only in the direction of data transfer to sink. In Figure 3, the cluster configuration angle is $\pi / 2$. If the composition angle exceeds $\pi$, data is transferred in the reverse direction, so only angles smaller than $\pi$ are considered.

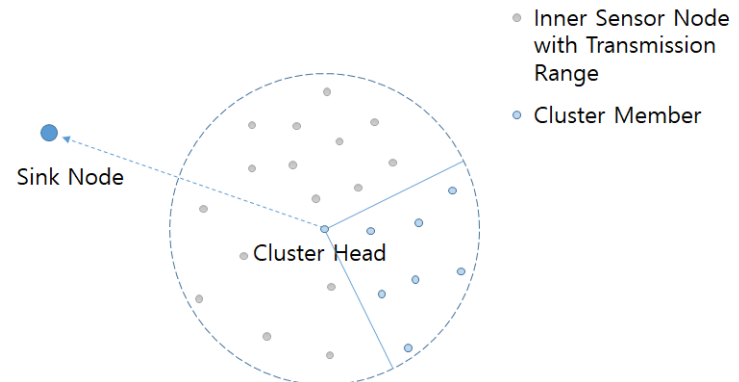

Fig 3. Cluster Configuration with Header and Member

The smaller the angle, the closer the header relation to the members becomes, the shorter the propagation path becomes. However, since the size of the cluster becomes smaller and the number of the header becomes larger, the suitability of the cluster configuration becomes lower. Instead, a cluster configuration of 1 hop more can be considered.

\section{SIMULATION AND RESULT}

In order to measure the performance of the proposed method, we compared the results by simulation based on C\#. The network size was 500 by 500 or 1000 by 1000 and the sink node was located at the top left $(0,0)$. The sensor nodes are randomly arranged from 500 to 1000 depending on the density, and the communication radius is 100 . The composition angle of the cluster members was measured from $\pi / 2$ to $\pi$. The parameter values used in the simulation are shown in Table 1.
Table 1: Parameters of simulation

\begin{tabular}{|l|l|}
\hline Parameter & Values \\
\hline \hline Network size & $500 \times 500,1000 \times 1000$ \\
\hline Number of sensor nodes & $500 \sim 10000$ \\
\hline Transmission radius(TR) & 100 \\
\hline Angle of cluster member & $\pi / 2 \sim \pi$ \\
\hline
\end{tabular}

In Figure 4(a), each sensor node selects a neighbor node closest to the sink node and transmits the sink node by hop-by-hop. This data does not include the distance information but is only the direction information. As shown in Figure 4(a), it can be seen that when each sensor node uses only the direction information, it selects and optimal forwarding node toward the sink node. Figure 4(b) shows the $\cos \theta$ values of neighbor nodes of the sensor node located at $(500,500)$. The node with the red bold line is the most optimal forwarding node with lowest value of $\cos \theta$.

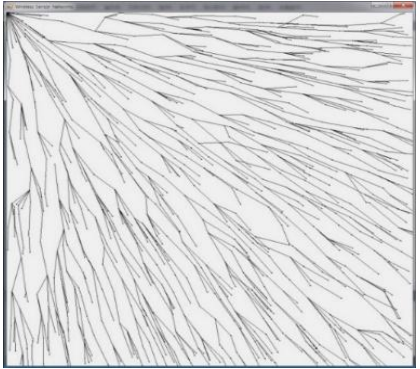

(a)

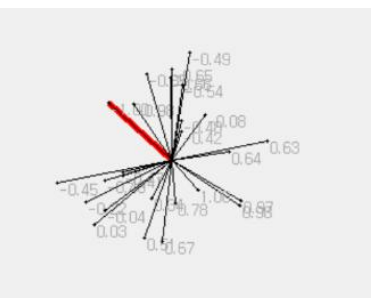

(b)
Fig 4. Relay node connection $($ NumNode $=1000, \mathrm{TR}=$ 100)

The sensor node is located at $(500,500)$ in the network 1000 by 1000 and the header possibility values of neighboring nodes are measured. Figure 5 shows the arrangement of the sensor nodes, and the header possibilities of the nodes. As shown in Figure 5, the closer to the sink node, the closer to the boundary of the communication radius, the larger the header probability value is. A sensor node connected by a red bold line is a header candidate node having the smallest value. Sensor nodes in the opposite direction to the data transfer to sink, that is, nodes with a positive header possibility value, returned a value of 0 . And backward sensor node is not applied when calculating the header possibility value.

Figure 6 shows the result of connecting each sensor node and neighbor node with the lowest possible header value to the forwarding node in the whole network. Figure 4 (a) only considers the closeness of the sink node direction, and Figure 6 considers direction and distance together. The connection between the sensor nodes does not consider the cluster but connects the node with the highest header probability among its neighbor nodes and connects to the sink by hop-by-hop. 


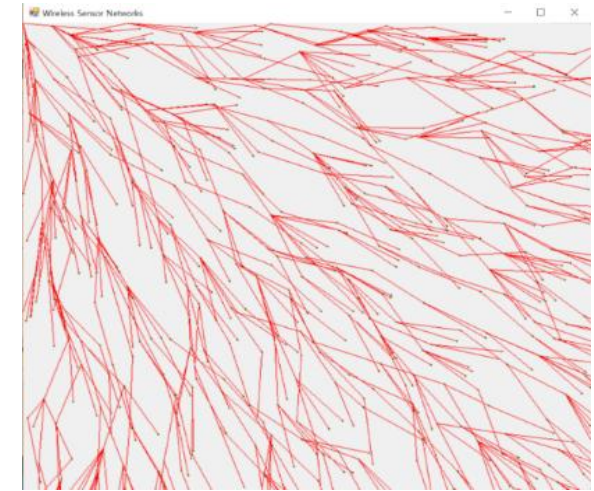

Fig 5. Connection with header possibility value (NumNode $=1000, \mathrm{TR}=100)$

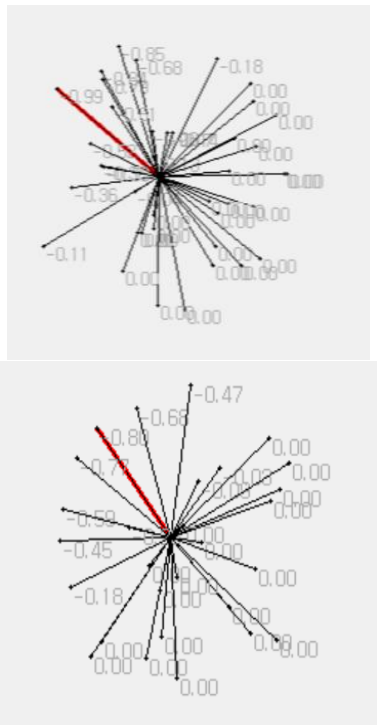

Fig 6. Header possibility value of Sensor Node located at $(500,500)$

Figure 7 shows the cluster configuration according to the angle allowed by the cluster header when selecting members. Figure 7 (a) shows the cluster configuration angle when the cluster configuration angle is $\pi$, and Figure 7 (b) shows the cluster configuration when it is $2 \pi / 3$.

Figure 8 compares the amount of energy consumed when sensor node located at $(500,500)$ in the network 500 by 500 transmits data 1 bit directly to the sink node and the amount of energy used to transmit data through the cluster header. When routing through the cluster header, the routing between the cluster headers is carried through the high header probability neighbor node.

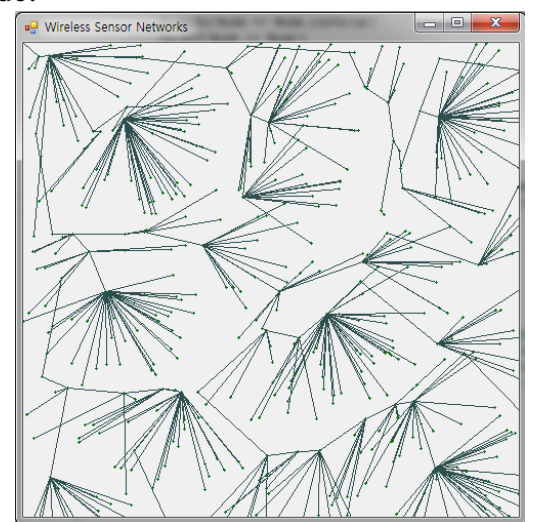

(a) Header angle : $\pi$
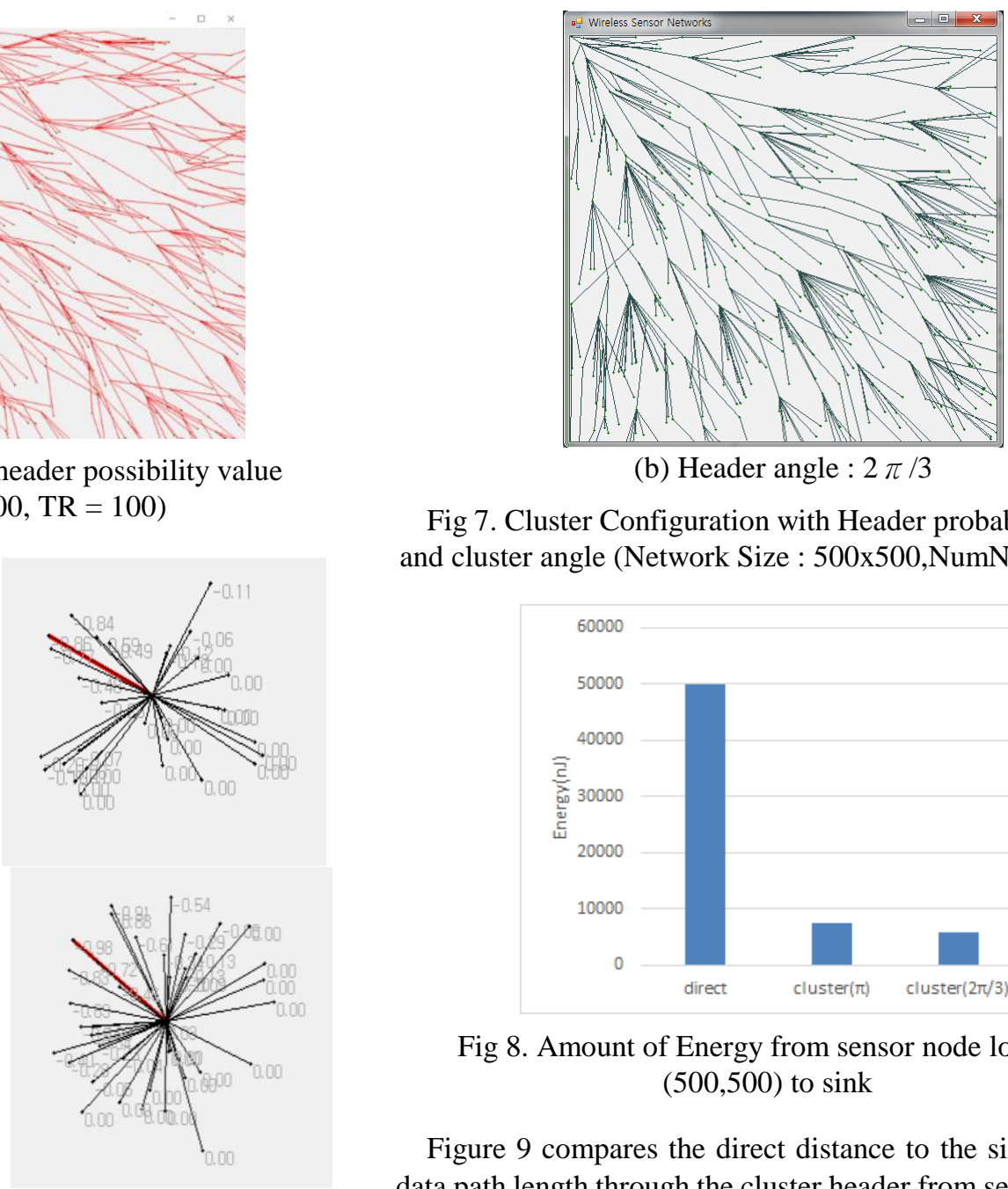

(b) Header angle : $2 \pi / 3$

Fig 7. Cluster Configuration with Header probability value and cluster angle (Network Size : 500x500,NumNode : 500)

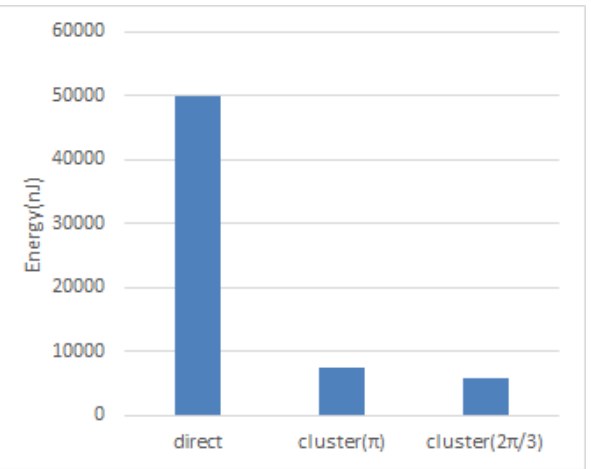

Fig 8. Amount of Energy from sensor node located in $(500,500)$ to sink

Figure 9 compares the direct distance to the sink and the data path length through the cluster header from sensor nodes located in $(500,500)$. We also compared the data transmission lengths of clusters with header angles. It can be seen that the direct transmission is the most optimal length, but the energy consumption shown in Figure 8 shows that it is difficult to apply in wireless sensor networks. When the cluster is formed without larger angle, it is seen that the transmission length is increased because it has to go through the cluster header in the opposite direction of the sink direction in the transmission radius. Clustering with header angle reduces reverse data transmission in the cluster and reduce the length of path to sink node. Figure 8 and 9 show that the proposed scheme is energy efficient and can reduce the path length to the sink.

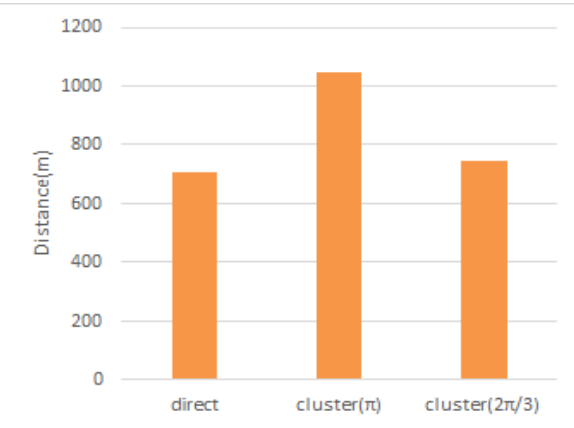

Fig 9. Distance of path from sensor node located in $(500,500)$ to sink 


\section{CONCLUSION}

In order to use energy efficiently in wireless sensor networks, multi-hop transmission is preferred by forming a cluster rather than directly transmitting data to a sink node. In the clustering technique, the energy efficiency may be changed depending on the selection method of the cluster headers and the configuration method of the cluster members.

In this paper, we analyze location elements closer to the sink by the qualification requirements of the cluster headers. In the transmission range, the probability value is set so that the node that is closest to the straight line to the sink and far from the distance becomes the header candidate. And sensor nodes in the direction of transmitting data to the sink node are selected as the cluster member by using the angle from the cluster header. Simulation results show that the proposed method can reduce the data transmission path while using efficient energy compared with direct transmission.

In the following paper, we try to analyze the ratio of distance elements and angular elements more precisely when calculating the cluster header probability value.

\section{ACKNOWLEDGMENT}

This research was supported by YongIn University.

\section{REFERENCES}

1. G. Anastasi, M. Conti, M. Francesco and A. Passarella, "Energy Conservation in Wireless Sensor Networks: A survey", Ad Hoc Networks, vol.7, no. 3, 2009 May, pp. 537-568. Available: https://doi.org/10.1016/j.adhoc.2008.06.003

2. Beom-Su Kim, HoSung Park, KyongHoon Kim, Daniel Godfrey and Ki-Il Kim, "A Survey on Real-Time Communications in Wireless Sensor Networks", Wireless Communications and Mobile Computing, 2017 October, pp. 1-14. Available: https://doi.org/10.1155/2017/1864847

3. Kemal Akkaya and Mohamed Younis, "A survey on routing protocols for wireless sensor networks", Ad Hoc Networks, vol.3, no. 3, 2005 May, pp. 325-349. Available: https://doi.org/10.1016/j.adhoc.2003.09.010

4. A.A. Abbasi and M. Younis, "A survey on clustering algorithms for wireless sensor networks", Computer Communications 30, vol.30, no. 14, 2007 October, pp. 2826-2841. Available: https://doi.org/10.1016/j.comcom.2007.05.024

5. Santar Pal Singh and S.C. Sharma, "A Survey on Cluster Based Routing Protocols in Wireless Sensor Networks", in International Conference on Advanced Computing Technologies and Applications, vol. 45, 2015, pp. 687-695. Available: https://doi.org/10.1016/j.procs.2015.03.133

6. Asim Zeb1, A. K. M. Muzahidul Islam1, Mahdi Zareei1, Ishtiak Al Mamoon2, Nafees Mansoor3 and Sabariah Baharun1, "Clustering Analysis in Wireless Sensor Networks: The Ambit of Performance Metrics and Schemes Taxonomy", International Journal of Distributed Sensor Networks, vol. 12, no. 7, 2016 July, pp. 1-24. Available: https://doi.org/10.1177/155014774979142

7. S. Pal Singh and S.C. Sharma, "A Survey on Cluster Based Routing Protocols in Wireless Sensor Networks", in International Conference on Advanced Computing Technologies and Applications(ICACTA), vol. 45, 2015, pp. 685-695. Available: https://doi.org/10.1016/j.procs.2015.03.133

8. W. B. Heinzelman, A. Chandrakasan and H. Balakrishanan, "An application-specific protocol architecture for wireless microsensor networks", IEEE Transactions on Wireless Communication, vol. 1, no. 4, 2002 October, pp. 660-670. Available: https://doi.org/10.1109/TWC.2002.804190

9. O. Younis and S. Fahmy, "HEED: A hybrid, energy-efficient, distributed clustering approach for ad hoc sensor networks", IEEE Transactions on Mobile Computing, vol. 3, no. 4, 2004, pp. 366-379. Available: https://doi.org/10.1109/TMC.2004.41

10. S. Lindsey and C. Raghavendra, "Pegasis: Power-efficient gathering in sensor information systems", in Proceedings of IEEE Aerospace
Conference, vol. 3, 2002 March, pp. 1125-1130. Available: https://doi.org/10.1109/AERO.2002.1035242

11. Y.Xu, J. Heidemann and D.Estrin, "Geography-informed Energy Conservation for Ad Hoc Routing “, in Proceeding of the 7th annual international conference on Mobile computing and networking, 2001, pp. 70-84. Available: https://doi.org/10.1145/381677.381685

12. H. O. Tan and I. Korpeoglu, "Power efficient data gathering and aggregation in wireless sensor networks", SIGMOD Record, vol. 32, no. 4, 2003 December, pp. 66-71. Available: https://doi.org/10.1145/959060.959072

13. Zhao Han, Jie Wu, Jie Zhang, Liefeng Liu and Kaiyun Tian, "A General Self-Organized Tree-Based Energy-Balance Routing Protocol for Wireless Sensor Network", IEEE Transactions on Nuclear Science, vol. 61, no. 2, 2014 April, pp. 732-740. Available: https://doi.org/10.1109/TNS.2014.2309351

14. EunHwa Kim, "Cluster Formation with Forwarding Direction at Multi-hop Clustering Model in a Wireless Sensor Networks", Advanced Science Letters, vol. 23, no. 10, 2017 October, pp. 10293-10297. Available: https://doi.org/10.1166/asl.2017.10438

15. EunHwa Kim, "Cluster Head Selection Elements in a Wireless Sensor Networks", in International Conference on Next Generation Computer and Information Technology (NGCIT2018), 2018 August.

\section{AUTHORS PROFILE}

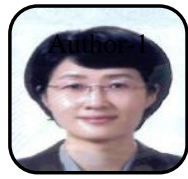

EunHwa Kim she is a BS degree in Computer Engineering at Kyoungpook National University in 1995. MS in Computer Engineering at Kyoungpook National University in 1999. Ph. D in Computer Engineering at Kyoungpook National University in 2008. Software engineer at Samsung Electronics from 1995 to 1996. Assistant Professor of Computer Science at Yongin University from 2012. 